\title{
A non-contact laser speckle sensor for the measurement of robotic tool speed
}

\author{
Thomas O.H. Charrett ${ }^{\mathrm{a}, *}$, Yashwanth K. Bandari ${ }^{\mathrm{b}}$, Florent Michel ${ }^{\mathrm{b}}$, Jialuo Ding ${ }^{\mathrm{b}}$, Stewart W. \\ Williams $^{\mathrm{b}}$, Ralph P. Tatam ${ }^{\mathrm{a}}$ \\ ${ }^{a}$ Centre for Engineering Photonics, Cranfield University, MK43 OAL, UK \\ ${ }^{b}$ Welding Engineering and Laser Processing Centre, Cranfield University, MK43 OAL, UK
}

\begin{abstract}
A non-contact speckle correlation sensor for the measurement of robotic tool speed is described that is capable of measuring the in-plane relative velocities between a robot end-effector and the workplace or other surface. The sensor performance has been assessed in the laboratory with sensor accuracies of $\pm 0.01 \mathrm{~mm} / \mathrm{s}$ over a $\pm 70 \mathrm{~mm} / \mathrm{s}$ velocity range. The effect of misalignment of the sensor on the robot was assessed for variation in both working distance and angular alignment with sensor accuracy maintained to within $0.025 \mathrm{~mm} / \mathrm{s}(<0.04 \%)$ over a working distance variation of $\pm 5 \mathrm{~mm}$ from the sensor design distance and $\pm 0.4 \mathrm{~mm} / \mathrm{s}(0.6 \%)$ for a misalignment of $5^{\circ}$. The sensor precision was found to be limited by the peak fitting accuracy used in the signal processing with peak errors of $\pm 0.34 \mathrm{~mm} / \mathrm{s}$. Finally an example of the sensor's application to robotic manufacturing is presented where the sensor was applied to tool speed measurement for path planning in the wire and arc additive manufacturing process using a KUKA KR150 L110/2 industrial robot.
\end{abstract}

Keywords: Laser speckle, Tool speed, Velocimetry, Robotic sensor,

\section{Introduction}

In many areas of manufacturing it is desirable to replace expensive Computer Numerical Control (CNC) systems with a robotic approach providing increased flexibility and lower costs. However, typical industrial robots have comparatively low mechanical stiffness[1] and are more prone to disturbances from process forces. In addition there can be significant deviations from the desired tool-path and tool-speed due to thermal and systematic errors in the kinematic model used to convert joint encoder positions to Cartesian end-effector position[2]. Hence, characterisation of the robot motion is of great importance in many manufacturing operations, for example, in many continuous machining or processing operations the feed rate or tool speed is critical to process quality[3]. External measurements systems such as laser trackers[4], iGPS[5], position sensitive detectors[6] or vision systems[7,8] can be used to track the motion of the robot end-effector. However, these methods of monitoring the motion also suffer limitations; vision

\footnotetext{
*Corresponding author

Email address: t.charrett@cranfield.ac.uk (Thomas O.H. Charrett)
} 
systems have limited update rates, methods using position-sensitive detectors[6] are limited to operating within a fixed plane, and laser scanners or laser interferometric guidance systems[4] are expensive and inflexible as the scanning system needs to be mounted externally to the robot and maintain a continuous line-of-sight and which may become obstructed.

This paper describes an alternative approach using laser speckle correlation sensing to make live measurements of the robotic tool speed and is intended to introduce the technique to researcher, demonstrate the achievable performance, and discuss potential challenges and limitations of the technique. In this approach the sensor is attached to the robot end-effector and measures the relative motion between the end-effector and work-piece by high speed processing of laser speckle patterns. Laser speckle patterns, named for their characteristic granular appearance, are formed when coherent light, such as the output from a laser, illuminates a surface with a roughness larger than the optical wavelength[9]. The translation and de-correlation of a speckle pattern are related to the illuminated objects translation, rotation, strain and surface roughness in a family of techniques termed laser speckle correlation that were first described in the late 1970s and early 1980s[10]. Although well understood, the techniques have only recently become viable for practical applications in manufacturing and robotics due to advances in camera and signal processing technology. This has led to renewed interest in the technique with researchers investigating new applications in robotics vehicle odometry[11, 12] and robotic positioning and stabilisation applications $[13,14]$.

Speckle correlation sensors have the potential to provide low-cost on-line measurement of tool speed and position and in this paper the design and signal processing of a speckle correlation sensor for tool speed measurement is described. The sensor performance is then assessed in section 3, including the achievable accuracy and precision, along with the sensitivity to misalignments and robot error motions. Finally, in section 4, an example application of speckle correlation sensing for a robotic manufacturing process is reported where the sensor was applied to tool speed measurement for path planning in the wire and arc additive manufacturing (WAAM) process $[15,16]$. This application shows the potential of the speckle correlation approach for real-time measurement of end-effector speed that could be applied in a wide range of other robotic manufacturing processes.

\section{Sensor design and signal processing}

The concept of the sensor is shown in Figure 1 a) where the sensor is attached to the robot end-effector and measures the relative in-plane translation between the robot/sensor and workpiece. The signal processing principle is shown in Figure $1 \mathrm{~b}$ ) where the two-dimensional normalised cross-correlation [17] between a reference speckle pattern and a newly acquired speckle pattern is computed. The offset of the peak from the centre of correlation image gives the shift of the speckle pattern. $\left(A_{x}, A_{y}\right)$ which can then be related to the $x$ and $y$ translations between the sensor and workpiece occurring between the images. It should be noted that the laser speckle patterns used by the sensor can be formed from a wide variety of surfaces as long as the surface is rough at the scale of the optical wavelength $(\sim 0.7 \mu \mathrm{m})$, i.e. diffusely reflecting . For the work here the workpiece surface is assumed to be metallic for example the build plate used in the WAAM process[15].

The prototype sensor used in this work (Figure 2) consisted of a fibre coupled diode laser source operating at $658 \mathrm{~nm}$ (FibreTec II FTEC2658-P60PA0, max output 60mW, operating output $0.6 \mathrm{~mW}$ ) delivered to the sensor head via an armoured fibre cable containing a single-mode optical fibre (Nufern PM-S630-HP). The output from the fibre is then shaped and focused to a waste at 
a)

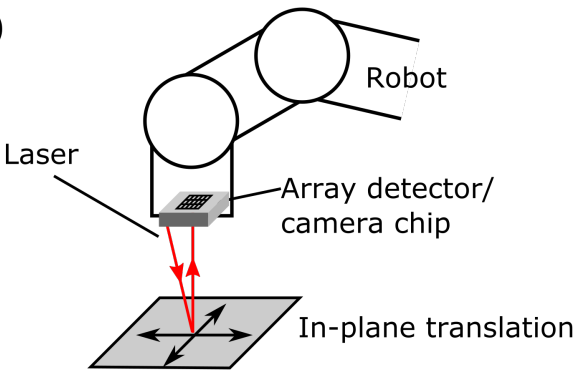

b)

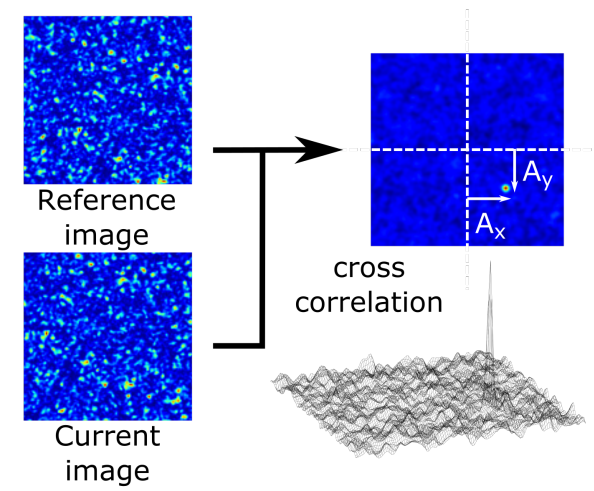

Figure 1: Sensor concept. In a) the sensor is attached to the robot end-effector to measure relative in-plane translation between the robot and workpiece. In $\mathrm{b}$ ) the signal processing principle is shown with the $2 \mathrm{D}$ cross-correlation between a reference and new speckle pattern computed with the peak giving the translation of the pattern $\left(A_{x}, A_{y}\right)$.

point $S$, via collimation and focusing lenses ( $\mathrm{f}=15 \mathrm{~mm}$ and $\mathrm{f}=50 \mathrm{~mm}$ ) contained in the lens assembly. The beam then expands to spot, $R$ of approximately $8 \mathrm{~mm}$ diameter on the workpiece. The resulting speckle patterns formed by scattering from the surface are recorded by a detector array, $D$, a high speed camera (Ximea MQ013CG-ON) operating at 500fps with exposure times of $200 \mu$ s and acquiring a region-of-interest of $512 \times 512$ pixels. A laser-line band-pass filter (Semrock FF01-655/40-25, 655nm centre wavelength, 40nm bandwidth, optical density $>5$ ) is mounted in front of the camera detector array to reduce ambient background light and prevent sensor blinding.

The detector array centre point, $D$, and beam waist, $S$ are aligned to lie within a common $x y$ plane at $z=150 \mathrm{~mm}$ from the workpiece, and are arranged symmetrically around the $z$-axis centred on the laser spot, $R$. This balanced-angle geometry offers several advantages: strong signal levels due to operating in the narrow scattering cone of the metallic surface around the specular reflection angle; and theoretically zero sensitivity to out-of-plane motion when $D$ and $S$ are located in the same $x y$ plane [18]. The distance $D S$ was kept small to create a compact sensor (diameter $50 \mathrm{~mm}$ ) and to reduce the angle between $S R D$ and minimise sensitivity changes due to working height variations [11] and surface gradients [19]. Whilst a co-linear system with the vectors $S R$ and $R D$ coincident would be optimum, the small angle geometry was chosen due to the reduced number of optical components required, and the simpler, more compact implementation. The components where mounted in a 3D printed polylactic acid plastic barrel mount 

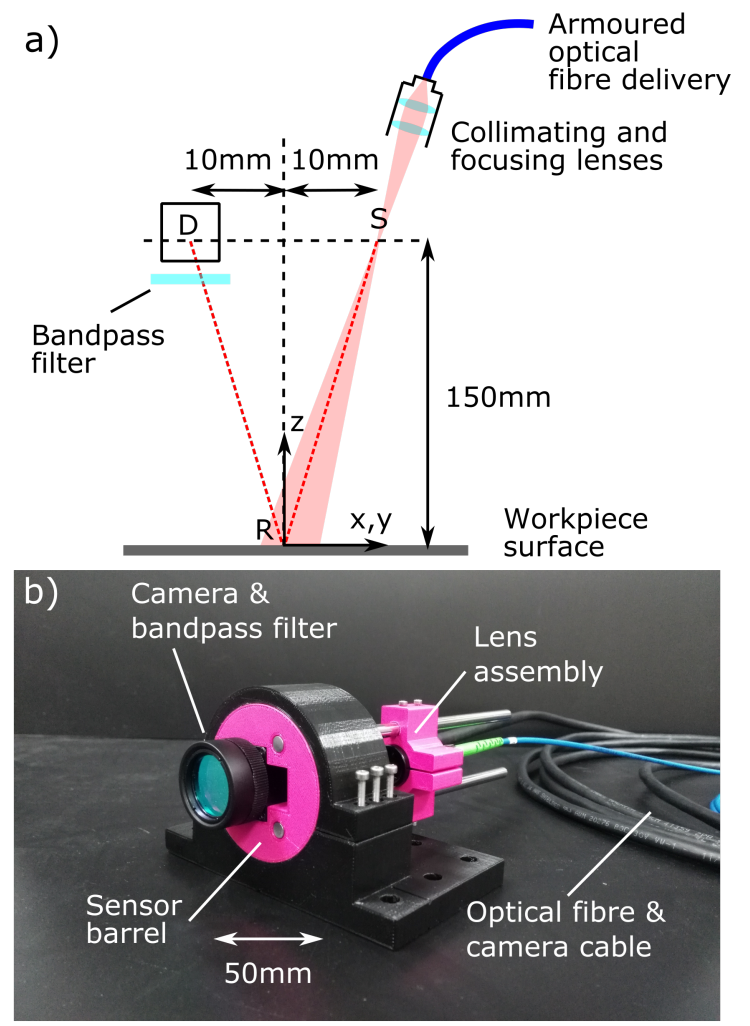

Figure 2: a) A schematic showing the balanced angle geometry used for the sensor with the beam focus and detector located in the same $x-y$ plane $150 \mathrm{~mm}$ from the workpiece surface and $b$ ) a photograph of the 3D printed sensor prototype in mount.

allowing the sensor to be rotated about the z-axis for setup and calibration purposes. It is important to note that an objective or 'lensless' speckle sensor was used here, with no imaging lenses in front of the camera chip. This approach has a number of advantages, in particular, the reduction in the number of optical components required, and the removal of the need for a small aperture used to increase speckle size which reduces the available light in subjective (imaged) speckle measurements [11].

\subsection{Signal processing}

The signal processing, shown in Figure $1 \mathrm{~b}$ ), consists of acquiring and processing frames in real time at high frame rates $(\sim 500 \mathrm{fps})$. Initially the first speckle pattern is stored as the reference with relative position initialised to $(0,0)$. The translation between each newly acquired frame and this reference image is then calculated via the computation of the 2D normalised cross-correlation [17] where the peak position gives the translation between the two images. This peak position is determined to sub-pixel accuracy using a three-point Gaussian fit [20]. Once the speckle shift with respect to the reference image has been found, this is converted to a real translation via the pre-calibrated scaling factors, discussed below, and the velocity can be found by differentiation with the previous position. This is in contrast to our previous work in 
speckle velocimetry for autonomous vehicle odometry $[12,11]$, which used correlation between consecutive frames to find the velocity. This process of correlating new images with a fixed reference, as described below, instead of sequential image pairs has several advantages. Firstly by integrating the total shift from the initial reference a better estimate of the robot position can be computed than is possible by integrating noisy velocity measurements derived from sequential image pairs. This allows for easier calibrations over longer distances, increasing the accuracy of the calibration. Additionally velocity bias errors due to pixel-locking [20], a cyclical biasing effect in the calculation of peak position for sub-pixel shifts in the correlation, are reduced due to averaging over several frames.

To allow larger translations before re-referencing is necessary, a larger reference speckle pattern is desirable hence a moving correlation window scheme is applied as shown in Figure 3. This allows a smaller correlation image size, enabling higher processing rates, and an increased velocity range whilst reducing the number of re-referencing operations and the accumulation of error in the integrated position used for calibration. In Figure 3 a) initially the reference and current image frame overlap the same region of the scattered speckle field and the correlation window (shown as the hatched rectangle) is centred in both frames. In Figure $3 \mathrm{~b}$ ) after a sensor translation, the reference image and current image are now offset with respect to each other in the scattered speckle field, hence to ensure maximum overlap between the correlation windows the windows are offset in opposite directions in the reference and current frame. This additional offset is then added to the peak shift found from the correlation to give the total shift between the two frames. In Figure $3 \mathrm{c}$ ) the sensor has reached the maximum translation where the correlation windows fully overlap, and the correlation windows now lie on opposite sides of the two images. For the next frame a re-referencing is performed as shown in Figure $3 \mathrm{~d}$ ). The previous frame is stored as the new reference with the position updated to reflect the new references offset. This process is then repeated as the total translation increases and this moving window scheme is applied in both the $x$ and $y$ directions simultaneously to account for shifts not aligned along image rows or columns. In this work a 512x512 pixel image size, and 128x128 pixel correlation window size was used allowing the processing to proceed at $500 \mathrm{fps}$. For the camera used this allowed re-referencing approximately every $1.2 \mathrm{~mm}$. The maximum velocity that can be measured is now limited by the size of correlation window and padding used in the calculation. Here a circular cross-correlation [20] was used for speed, allowing maximum speckle translations per frame of \pm 64 pixels for the $128 \times 128$ correlation window used. For the geometry shown in Figure 2 together with a 500fps processing rate and $4.8 \mu \mathrm{m}$ pixel size this corresponds to a maximum velocity of $75 \mathrm{~mm} / \mathrm{s}$. Higher velocity ranges should be possible using larger pixel sizes, higher frame rates or larger correlation window sizes.

\subsection{Calibration}

The sensor is calibrated by translating the sensor in the $x$ direction by a known distance $a_{x}$ while recording the resulting integrated speckle shift $\left(A_{x x}, A_{y x}\right)$. Here the first subscript denotes the component of the speckle shift and the second the robot/sensor translation component. This is then repeated for a translation in the $y$ direction, $a_{y}$ giving values for the speckle shift $A=$ $\left(A_{x y}, A_{y y}\right)$. These values are then used to find the translational scaling factor matrix, $T$, relating 
a)

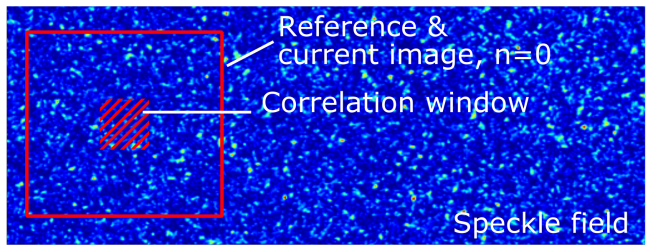

b)

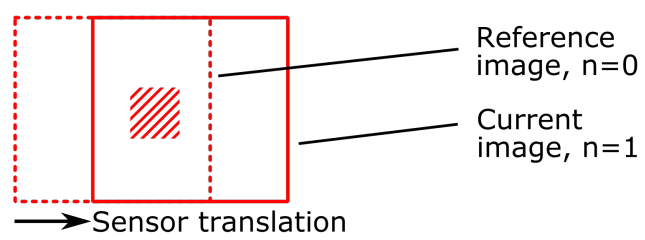

c)

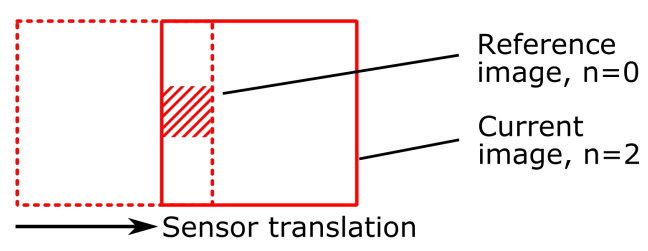

d)

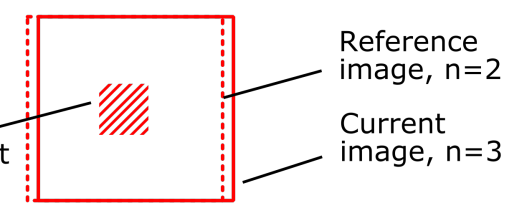

Figure 3: Overview of windowing scheme used in signal processing. In a) the initial reference image and current image overlap in the speckle field and the correlation is performed over the hatched window shown in both images. In b) and c) the windows are shifted in opposite directions in the reference and the current frame as the sensor translates. In d) a re-reference is performed where the previous frame is stored as the reference and the correlation windows reset to the centre. The total translation is stored internally and the process repeats.

speckle shift to robot translation by:

$$
\begin{gathered}
A=T a \\
{\left[\begin{array}{l}
A_{x} \\
A_{y}
\end{array}\right]=\left[\begin{array}{ll}
T_{x x} & T_{x y} \\
T_{y x} & T_{y y}
\end{array}\right]\left[\begin{array}{l}
a_{x} \\
a_{y}
\end{array}\right]}
\end{gathered}
$$

with the elements of $T$ given by:

$$
\begin{array}{ll}
T_{x x}=A_{x x} / a_{x} & T_{y x}=A_{y x} / a_{x} \\
T_{x y}=A_{y x} / a_{y} & T_{y y}=A_{y y} / a_{y}
\end{array}
$$

Values for these scaling factors can also be calculated from the sensor geometry $[18,19]$ although calibration is preferred to account for small variations in focus position and alignment. The values calculated in this way are then used in the signal processing to determine the translation 
Table 1: Sensor theoretical and calibrated scaling factors using the notation from [19]. Here the 1st subscript refers to the speckle shift component and the 2 nd to the component of translation. i.e. $T_{x y}$ is $x$ speckle shift from $y$ translation.

\begin{tabular}{c|c|c}
\hline Scaling factor & Theoretical values & Calibrated values \\
\hline$T_{x x}$ & 2.0 & 1.9916 \\
$T_{y x}$ & 0.0 & 0.0041 \\
$T_{x y}$ & 0.0 & 0.0036 \\
$T_{y y}$ & 2.0 & 1.9912 \\
\hline
\end{tabular}

$\left(a_{x}, a_{y}\right)$ from a measured speckle shift $\left(A_{x}, A_{y}\right)$ via:

$$
\begin{aligned}
& a_{x}=\frac{\left(A_{x} T_{y y}-A_{y} T_{x y}\right)}{\left(T_{x x} T_{y y}-T_{x y} T_{y x}\right)} \\
& a_{y}=\frac{\left(A_{y} T_{x x}-A_{x} T_{y x}\right)}{\left(T_{x x} T_{y y}-T_{x y} T_{y x}\right)}
\end{aligned}
$$

This process is performed using the integrated speckle shift obtained from the moving window scheme as described above, and allows the calibration to be performed to higher accuracy over larger translations than would otherwise be possible if the speckle shift was limited to the sensor size, corresponding to a translation of only $\sim 3 \mathrm{~mm}$. The limit of the achievable accuracy in the measured velocity for a given calibration distance and translation accuracy can be approximated for the simple case of no cross-sensitivity between the $x$ and $y$ components of speckle shift and translation (see appendix Appendix A) as:

$$
\frac{\Delta v}{v}=\frac{1}{(1 \pm \Delta a / a)}-1
$$

Where $v$ and $\Delta v$ are the velocity and velocity bias error, and $a$ and $\Delta a$ are the applied translation and accuracy of the translation used for calibration. For a typical calibration distance of 200 $\mathrm{mm}$ and industrial robot accuracy of $0.1 \mathrm{~mm}$ the scaling factors can be found to a precision of $\sim 3$ decimal places, corresponding to a velocity accuracy limit of $0.035 \mathrm{~mm} / \mathrm{s}$ at $70 \mathrm{~mm} / \mathrm{s}$. If the calibration is performed on precision translation stages accurate to $5 \mu \mathrm{m}$ this can be improved to a theoretical limit of $0.0017 \mathrm{~mm} / \mathrm{s}$.

\section{Laboratory characterisation of the sensor}

To characterise the performance of the sensor accuracy and sensitivity to working height changes, the sensor was mounted above a cast aluminium tooling plate attached to a 6 degreeof-freedom positioning stage (ALIO Hybrid-Hexapod AI-HYBRID-HEX-60XY-15Z-56R) as shown in Figure 4. This allowed controlled $x$ and $y$ translations at velocities up to $500 \mathrm{~mm} / \mathrm{s}$ with a translational accuracy of $<4 \mu \mathrm{m}$ and repeatability $<3 \mu \mathrm{m}$ together with the ability to adjust the working distance and tilts $\left(\theta_{x}, \theta_{y}\right)$.

Initially the sensor is calibrated using the procedure described in section 2 above and the theoretical scaling factors together with the calibrated values are shown in Table 1. The slight variation is typically due to the geometry tolerances in the source and camera position, working distance and in the positioning of the laser focus, $S$. The sensor accuracy and precision could then be assessed by making a series of controlled moves of the aluminium sheet on the stage. 


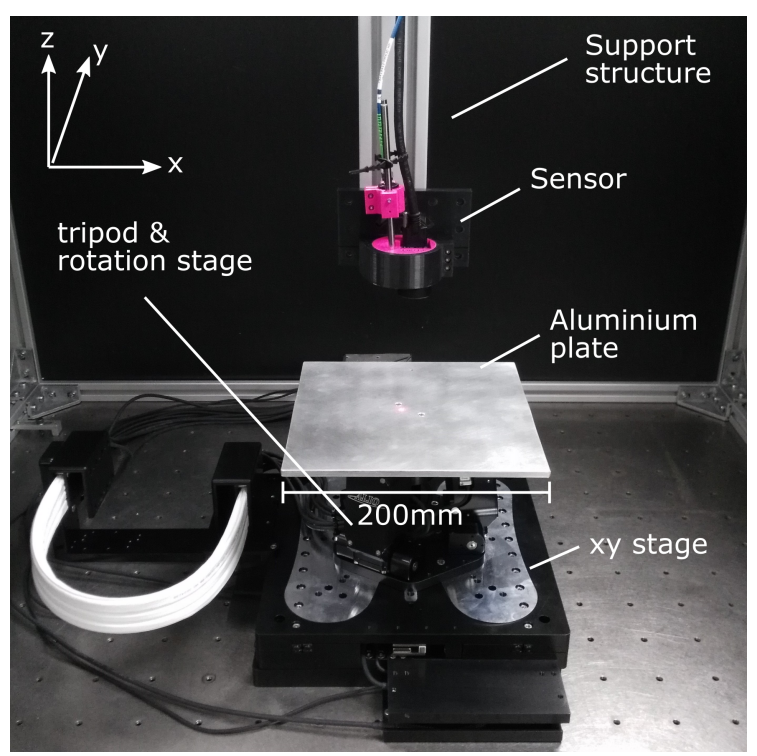

Figure 4: Experimental setup for laboratory measurements.

\subsection{Sensor linearity and accuracy}

Initially the sensor accuracy (bias error) and linearity was assessed for both measurements of the $v_{x}$ and $v_{y}$ velocity components with the results shown in figures 5 and 6 . Here the aluminium surface was translated by $200 \mathrm{~mm}$ at varying stage velocities between -70 and $+70 \mathrm{~mm} / \mathrm{s}$. The mean measured velocity was then calculated from 1000 samples ( 2 seconds) of the constant velocity period after the initial stage acceleration was completed.

Figure 5 (a) shows the results for a motion in the $x$ direction, with the red crosses showing the measured $v_{x}$ velocity component, the blue circles the $v_{y}$ component, and the dashed lines indicate the expected values. The equivalent results are shown in Figure 5 (b) for a motion in the $y$ direction. and the inset axes show the range of $\pm 10 \mathrm{~mm} / \mathrm{s}$ expanded. It can be seen that the sensor output is in good agreement with the expected values with minimal cross-talk between the two components of velocity.

To better assess the sensor accuracy, the remaining error, i.e. the mean measured velocity minus the mean stage velocity is shown in Figure 6. From these results it can be seen that the sensor accuracy is high with a maximum error of $\pm 0.01 \mathrm{~mm} / \mathrm{s}$ in mean recorded velocity over the full range $\pm 70 \mathrm{~mm} / \mathrm{s}$. Over the lower velocity ranges the error is also reduced further to $<0.004$ $\mathrm{mm} / \mathrm{s}$. There is some slight cross-talk present with $v_{x}$ motion leading to slight $v_{y}$ velocities being recorded and vice-versa, as indicated by the approximately linear form of the $v_{y}$ (blue) line in Figure 6 (a) and the $v_{x}$ (red) line in Figure 6 (b). However this is less than $0.005 \mathrm{~mm} / \mathrm{s}$ and is smaller than the bias error in the main travel direction. The remaining error in the main travel direction component of velocity has a more complex form possible due to pixel-locking effects [20] at certain velocities, the blurring of speckles at higher speeds or slight changes in scaling factors across the camera image together with the use of the moving window signal processing scheme shown in Figure 3. However it is clear that any remaining bias error is small enough to be considered insignificant for many applications. 
(a) sensor linearity for $v_{x}$ motion

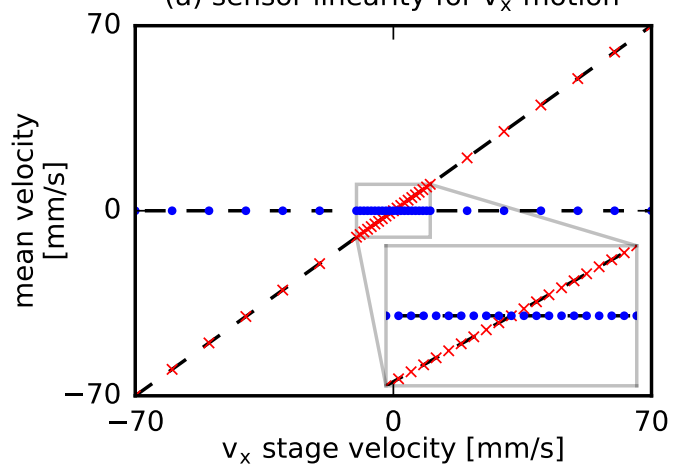

(b) sensor linearity for $v_{y}$ motion

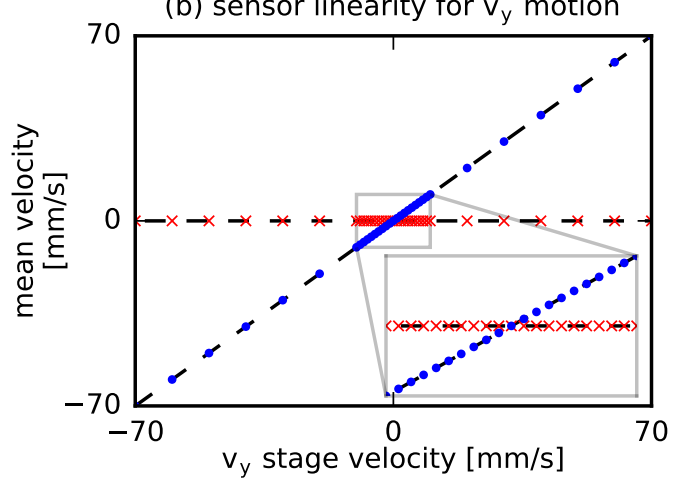

Figure 5: Measurements of sensor linearity for (a) motion in the $x$ direction and (b) motion in the $y$ direction. Here the mean sensor velocity ( 1000 samples $=2$ seconds) is shown plotted against the translation stage velocity, with red crosses representing the sensor $v_{x}$ component and blue dots the sensor $v_{y}$ component. The inset shows the range $\pm 10 \mathrm{~mm} / \mathrm{s}$. The dashed lines are for reference and show the zero error lines for the $x$ and $y$ components.

\subsection{Sensitivity to misalignments and working height changes}

A more important consideration is additional bias errors introduced by sensor miscalibration due to working height changes or angular misalignments of the sensor orientation during installation and operation. The accuracy of the sensor is dependent upon having the correctly calibrated scaling factors, which in turn are dependent upon the sensing geometry. Hence any change in working distance or orientation may lead to an increase in this bias error and potentially crossdependency between the two orthogonal velocity components.

To assess the sensitivity of the sensor to working height change the surface position was varied in the $z$ direction between -5 and $+5 \mathrm{~mm}$ from the calibration point. The results are shown in Figure 7, where (a) shows the resulting sensor accuracy/crosstalk when an $x$-component of velocity is applied to the stage with the top axes showing the resulting bias error in the measured $v_{x}$ component and the lower axes showing the crosstalk into the measured $v_{y}$ velocity component. The changes as the sensor working distance is varied by $\pm 5 \mathrm{~mm}$ from the design point are shown by the progressively darker lines. Similarly Figure 7 (b) shows the results when a $y$-component of velocity is applied to the stage. It can be seen that bias errors will increase predominantly for the $v_{x}$ velocity component with working height change. This is due to the balanced angle geometry used in this work where by a change in working distance will cause the translation 
(a) sensor accuracy/crosstalk for $\mathrm{v}_{\mathrm{x}}$ motion

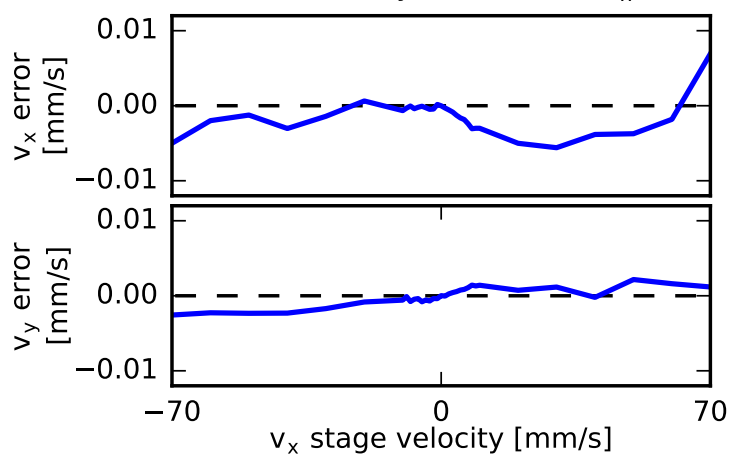

(b) sensor accuracy/crosstalk for $v_{y}$ motion

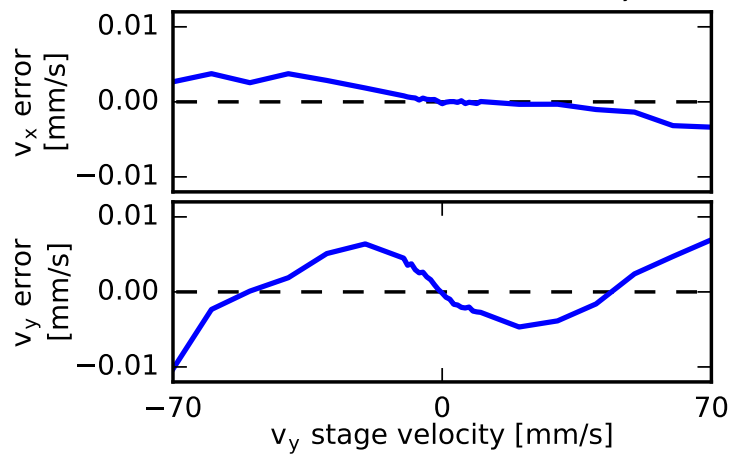

Figure 6: Measurements of sensor accuracy for (a) motion in the $x$ direction and (b) motion in the $y$ direction. The error in the measured $v_{x}$ and $v_{y}$ velocity components are shown on different axis for clarity and the zero error line is shown by the dashed line for reference.

of the spot relative to the sensor, increasing the change in scaling factors associated with that component, in this case the $T_{x x}$ scaling factor. However as the illumination and observation angles are small and close to the surface normal the changes in scaling factors are also small and the sensor accuracy can be maintained to within $0.025 \mathrm{~mm} / \mathrm{s}(<0.4 \%)$ over a working distance variation of $\pm 5 \mathrm{~mm}$ from the sensor design distance over a $\pm 70 \mathrm{~mm} / \mathrm{s}$ velocity range, hence the sensor can be considered robust to working height changes or misalignments.

Similarly if the sensor is misaligned during installation or use, by a change in the relative orientation between the sensor and surface, i.e. a tilt about the $x$ or $y$-axis ( $\theta_{x}$ and $\theta_{y}$ respectively) then the scaling factors and bias error may also change. In-plane rotations about the $z$-axis, $\theta_{z}$ will not cause a change in the scaling factors only a change in the transformation from sensor to world coordinate frames. Such tilts may be anticipated for sensors mounted on robotic endeffectors and orientation accuracy errors of up to $\pm 0.7^{\circ}$ for $\theta_{x}$ and $\theta_{y}$ have been reported for uncalibrated robots $[21,22]$, although this can be reduced to $<0.02^{\circ}$ by calibration[21]. To assess the sensitivity of the sensor to such angular changes the surface orientation was changed by $\pm 5.0^{\circ}$ and the sensor accuracy measured as before. The results are shown in Figure 8 and Figure 9 for angular changes in the $\theta_{x}$ and $\theta_{y}$ tilt angles respectively. As before parts a) and b) show the resulting sensor accuracy/crosstalk when an $x$ and $y$ component of velocity are applied to the stage respectively and different tilts between $\pm 5^{\circ}$ are represented by progressively darker 
(a) sensor accuracy for $\mathrm{v}_{\mathrm{x}}$ motion

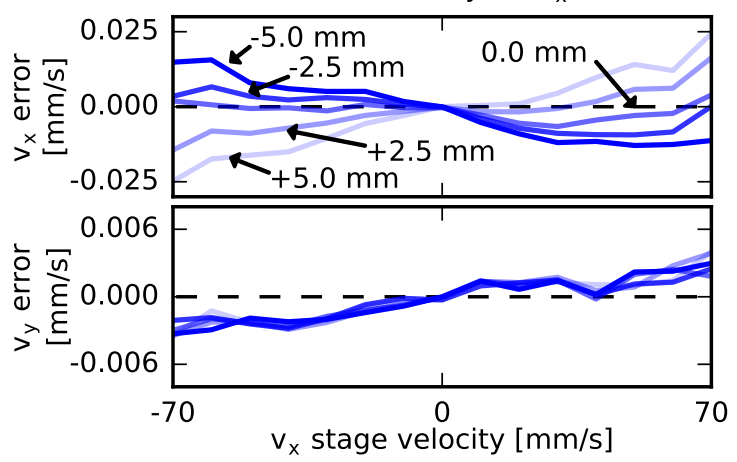

(b) Sensor accuracy for $v_{y}$ motion

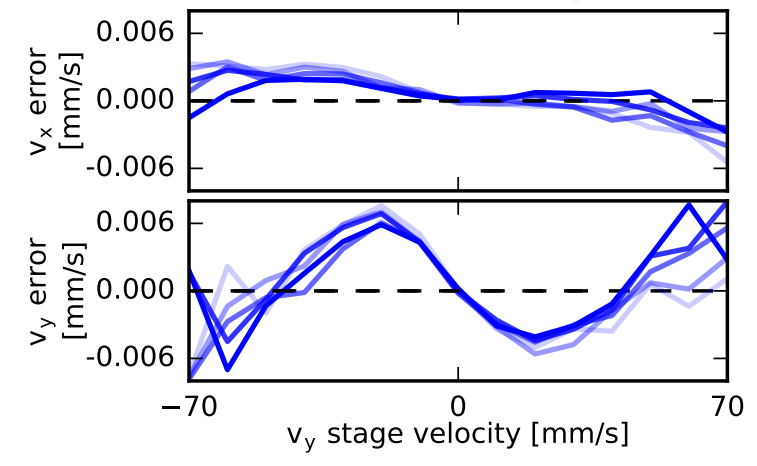

Figure 7: The sensor accuracy variation when the working distance is varied by $\pm 5 \mathrm{~mm}$ from the calibration distance for (a) motion in the $x$ direction and (b) motion in the $y$ direction where the accuracy/crosstalk in the measured $v_{x}$ and $v_{y}$ velocity components are shown on different axis for clarity. Working distances varied by $\pm 5 \mathrm{~mm}$ from the calibration distance are shown by progressively darker lines, as labelled in (a).

lines.

The effect of tilting the sensor is more pronounced than for a variation in working distance, with Figure 8 a) showing crosstalk into the $v_{y}$ velocity component from an applied $v_{x}$ motion increasing to a maximum $\pm 0.4 \mathrm{~mm} / \mathrm{s}$ when the sensors is tilted about $\theta_{x}$. The bias error/crosstalk remains lower for the other components at less than $\pm 0.05 \mathrm{~mm} / \mathrm{s}$. The results are similar for tilts about the $\theta_{y}$ angle as shown in Figure 9 with the bias error in the $v_{x}$ velocity component being most effected, again increasing to approximately $\pm 0.4 \mathrm{~mm} / \mathrm{s}$ for a $5^{\circ}$ tilt. However as can be seen in Figure 8 a) and Figure 9 a) the magnitude of this error is linear with both angle and velocity, hence for the expected tilts of $\pm 0.7^{\circ}$ reported for uncalibrated robots[21] this would be reduced to around $\pm 0.06 \mathrm{~mm} / \mathrm{s}$ at $70 \mathrm{~mm} / \mathrm{s}$ or $0.08 \%$.

\subsection{Sensitivity to other motion components}

In addition to bias errors caused by misalignments or changes in working distance or orientation the sensor will also be sensitive to other motion components such as tilts, or out-plane velocities. The sensitivity to these other motion components can be broken down into two effects. The first is the measurement of real velocities associated with the sensors offset from a centre of rotation. As the speckle correlation sensor is measuring the relative velocity at a point 
(a) sensor accuracy for $v_{x}$ motion

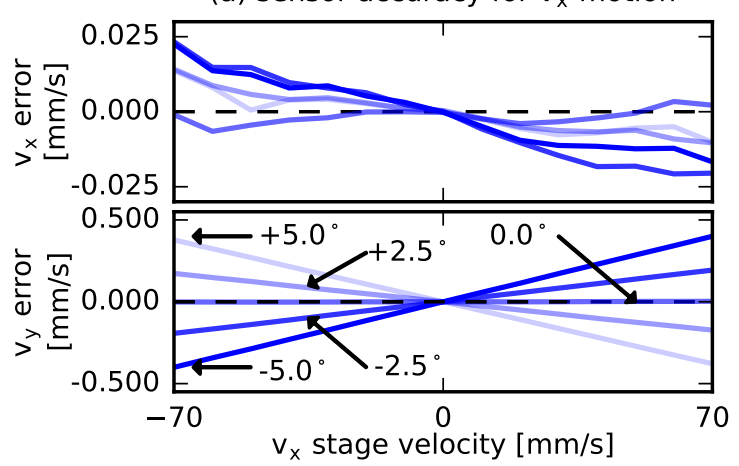

(b) Sensor accuracy for $v_{y}$ motion

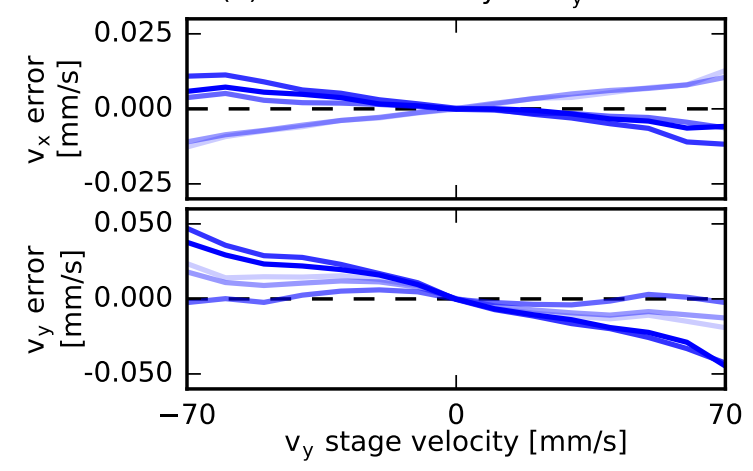

Figure 8: The sensor accuracy variation when the $\theta_{x}$ is varied by $\pm 5^{\circ}$ from the calibration distance for (a) motion in the $x$ direction and (b) motion in the $y$ direction where the accuracy/crosstalk in the measured $v_{x}$ and $v_{y}$ velocity components are shown on different axis for clarity. Results for $\theta_{x}$ varied between $\pm 5^{\circ}$ from the calibration point are shown by progressively darker lines, as labelled in (a).

on the surface defined by the laser spot location, any yawing or tilting motion will result in an additional translation depending upon the rotation angle and the offset from the centre of rotation. The second is speckle shift due to relative surface motions other than in-plane translations (in-plane-rotation, out-of-plane translations and tilts). These motions will cause an instantaneous translation of the speckle pattern occurring only for the duration of the error motion and will be recorded as spurious in-plane velocities. For oscillatory motions this will reduce the precision of the sensor, however ongoing velocities will add a bias error for the duration of the motion. This is different to the change in the accuracy associated with working distance or orientation miscalibration (section 3.2) that results in an on-going bias in the measurement of velocity even when the error motion is no longer occurring.

Yawing motion (rotation about the vertical z-axis) will result in a rotation of the speckle pattern about the laser spot for the sensing geometry used, plus an additional translation due to the offset of the laser point from the centre of rotation, hence to avoid magnification of these translations and the recording of these additional in-plane velocities the laser spot and tool centre point (TCP) should ideally be co-located.

Out-of-plane translations caused by either a step change in position or an ongoing out-ofplane velocity will cause small in-plane translations of the speckle pattern that will be recorded 
(a) sensor accuracy for $v_{x}$ motion

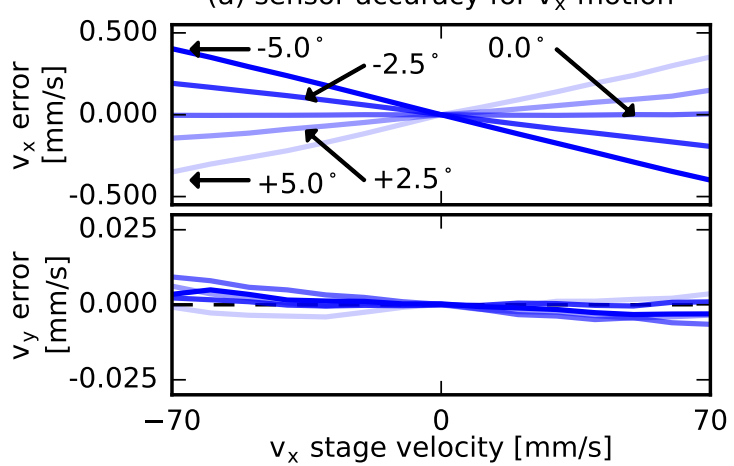

(b) Sensor accuracy for $v_{y}$ motion

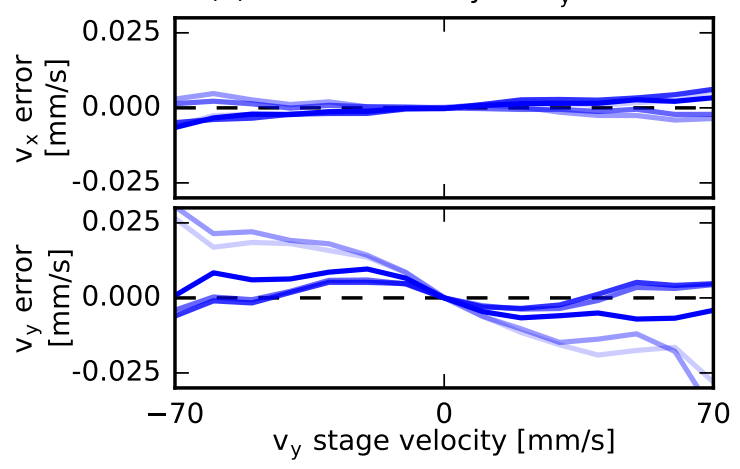

Figure 9: The sensor accuracy variation when the $\theta_{y}$ is varied by $\pm 5^{\circ}$ from the calibration distance for (a) motion in the $x$ direction and (b) motion in the $y$ direction where the accuracy/crosstalk in the measured $v_{x}$ and $v_{y}$ velocity components are shown on different axis for clarity. Results for $\theta_{y}$ varied between $\pm 5^{\circ}$ from the calibration point are shown by progressively darker lines, as labelled in (a).

as in-plane velocity components. For the 'balanced angle' geometry shown in Figure 2 the sensitivity to out-of-plane translations should theoretically be zero, however to assess the influence of out-of-plane motion on the sensor, speckle images were recorded before and after a $\pm 1 \mathrm{~mm}$ translation in the $z$-direction was applied to the stage. To reduce the influence of vibrations during the motion influencing the result, the stage was allowed to settle before the images were acquired and the speckle shift between the two frames found by normalised cross-correlation. The results are shown in Table 2 as translational scaling factors $T_{x z}$ and $T_{y z}$ which relate the speckle shift $\left(A_{x}, A_{y}\right)$ to the out-of-plane motion $a_{z}$ via:

$$
T_{x z}=A_{x} / a_{z} \quad T_{y z}=A_{y} / a_{z}
$$

This speckle shift will be converted to an in-plane translation via equation 4. Based upon the results shown in Table 2, an out-of-plane motion will result in a velocity error of $\left(v_{x}, v_{y}\right)=$ $(-0.004,+0.002) \mathrm{mm} / \mathrm{s}$ per $1 \mathrm{~mm} / \mathrm{s}$ velocity component in the $z$-direction.

Tilt errors (rotations about the $x$ and $y$ axes) cause both velocities associated with translations about the centre of rotation and erroneous velocities due to the surface rotation. The sensitivity to tilts about the laser spot was assessed as above with a rotations between $\pm 0.1^{\circ}$ applied to the 
Table 2: Sensor theoretical and calibrated scaling factors for sensitivity to error motions.

\begin{tabular}{c|c|c}
\hline Scaling factor & Theoretical values & Measured values \\
\hline$T_{x z}[-]$ & 0 & 0.0085 \\
$T_{y z}[-]$ & 0 & 0.0036 \\
$R_{x \theta x}\left[\mu \mathrm{m} /{ }^{\circ}\right]$ & 0 & 50 \\
$R_{y \theta x}\left[\mu \mathrm{m} /{ }^{\circ}\right]$ & 5259 & 5397 \\
& & \\
$R_{x \theta y}\left[\mu \mathrm{m} /{ }^{\circ}\right]$ & 5236 & 5798 \\
$R_{y \theta y}\left[\mu \mathrm{m} /{ }^{\circ}\right]$ & 0 & -65
\end{tabular}

stage. The results are shown in Table 2 as the rotational scaling factors in units of $\mu \mathrm{m} /{ }^{\circ}$ defined by:

$$
\begin{array}{ll}
R_{x \theta x}=A_{x} / \theta_{x} & R_{y \theta x}=A_{y} / \theta_{x} \\
R_{x \theta y}=A_{x} / \theta_{y} & R_{y \theta y}=A_{y} / \theta_{y}
\end{array}
$$

Although these numbers seem large the expected angular motion between two consecutive frames can be expected to be very small for example for a joint rotation of $90^{\circ}$ an angular error motion of $0.15^{\circ}$ may be expected [22], if this occurs over a 1 second period $\left(0.0003^{\circ}\right.$ between consecutive frames) this would correspond to a speckle shift of $\sim 1.7 \mu \mathrm{m}$ and a velocity error of $\sim 0.4 \mathrm{~mm} / \mathrm{s}$

\subsection{Sensor precision}

The measurement precision or repeatability of the sensor can be estimated by using the motion stage setup described above to simulate a motion and subtracting the stage position feedback from the sensor data recorded simultaneously. However although this will remove stage velocity fluctuations, other 'real' signals such as vibrations affecting only the sensor, and stage error motions such as tilts and deviations from flatness and straightness will still be recorded by the sensor as spurious velocity components. To provide a visual confirmation of the sensors performance and an estimate of the sensor repeatability, under a range of continuously varying $v_{x}$ and $v_{y}$ velocity components the stages where programmed to move in a circular motion at a constant travel speed. The results of this test are shown in Figure 10 for a travel speed of $1 \mathrm{~mm} / \mathrm{s}$ and Figure 11 for a travel speed of $70 \mathrm{~mm} / \mathrm{s}$. In these figures the top plot shows the measured $v_{x}$ and $v_{y}$ components plotted against time. Initially the stage travels at a constant velocity along the $y$-axis until the radius of the circle is the stage then pauses, before beginning the circular path which is repeated three times, finally the stage returns to the centre of the circle. The lower plots show the calculated velocity error found by subtraction of the stage encoder velocity feedback. This is calculated internally in the stage controller by differentiation of the position encoder feedback and all internal filtering in the controller was disabled for this test. The stage encoder data was then mapped to the sensor time samples using linear interpolation, before subtraction from the sensor data to give the velocity error shown.

It can be seen that the sensor resolves the sinusoidal velocity profiles well, with the remaining peak velocity error of $\pm 0.34 \mathrm{~mm} / \mathrm{s}$ at $1 \mathrm{~mm} / \mathrm{s}$ and $\pm 2.28 \mathrm{~mm} / \mathrm{s}$ at $70 \mathrm{~mm} / \mathrm{s}$. This difference can be attributed to increased vibrations at higher stage velocities, and it should be noted that for this system, a $2.28 \mathrm{~mm} / \mathrm{s}$ velocity error corresponds to a translation of only $4 \mu \mathrm{m}$ for the inter-frame 
time, of 2 ms used. Similarly the velocity error excluding peaks in Figure 10 is around \pm 0.15 $\mathrm{mm} / \mathrm{s}$ which corresponds to a position error of $0.3 \mu \mathrm{m}$ or 0.06 pixels which agrees well with previously reported values of between $1 / 10$ and $1 / 20$ of a pixel for the random error associated with peak fitting in similar correlation based techniques[20].

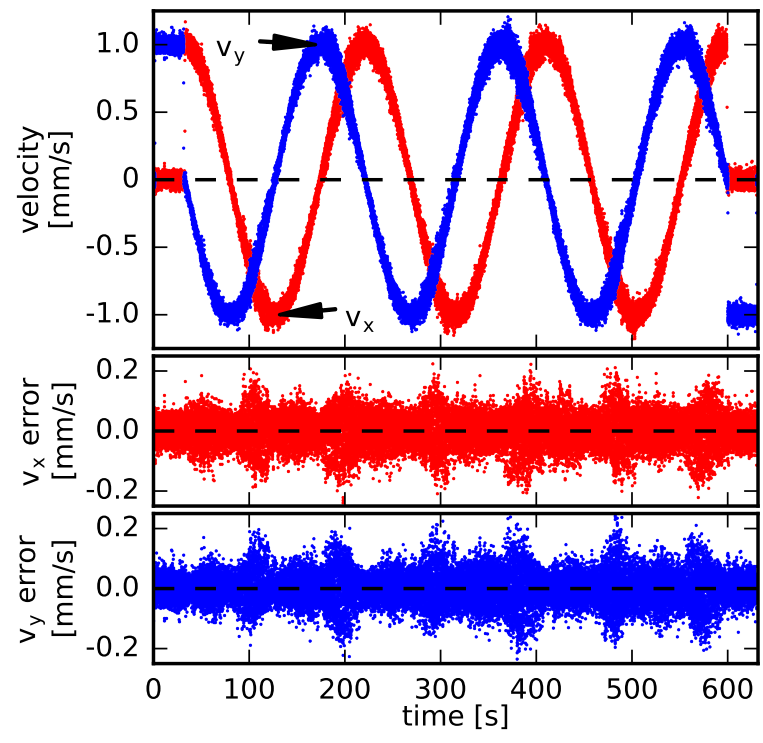

Figure 10: Circular path test at $1 \mathrm{~mm} / \mathrm{s}$ travel speed. The top plot shows the recorded sensor velocity components (red $v_{x}$, blue $-v_{y}$ ) and the bottom plots show the velocity component error referenced to stage encoder feedback.

\section{Example application of speckle velocimetry to robotic manufacturing}

The speckle velocimetry sensor described previously was applied for robot path characterisation of a KUKA KR150 L110/2 industrial robot used for robotic wire and arc additive manufacturing (WAAM). WAAM is an additive manufacturing process using a combination of an electric arc as the heat source and wire as feedstock [15] together with motion provided either by robotic systems or computer numerical controlled gantries. The use of robotic systems is desirable due to their increased flexibility, ease of integration with other processes and ability to produce larger parts however the increased deviations in tool path and tool speed can potentially limit the quality of the parts built. For example, an uncompensated reduction in tool speed will lead to increased material deposition leading to uneven top surface profiles and increased wall width [16] together with an associated increase in material usage and in the finishing machining required. Here the speckle sensor, described above was used to measure deviations of the real tool speed from commanded speed during different wall building strategies. Figure 12 a) shows the speckle sensor mounted on the robot, with the beam spot located at the tool centre-point (TCP) the welding torch position. This prevents the measurement of additional velocity due to robot yawing motion (rotation around the vertical axis) and sensor offset from the TCP. In Figure $12 \mathrm{~b}$ ) an example of oscillatory wall building path is shown where the robot was programmed to maintain a constant tool speed of $7 \mathrm{~mm} / \mathrm{s}$. An example result is shown in Figure 13, which shows 


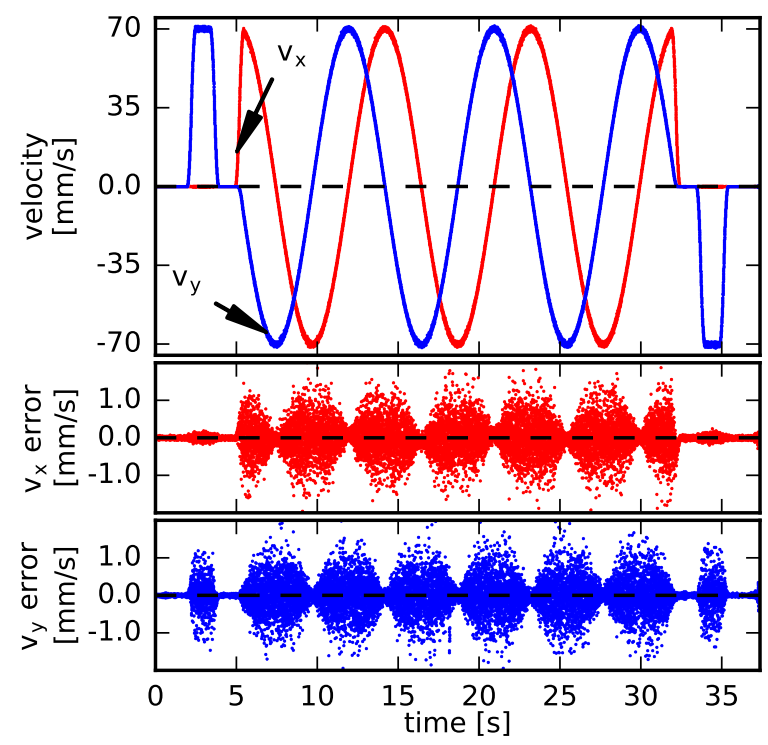

Figure 11: Circular path test at $70 \mathrm{~mm} / \mathrm{s}$ travel speed. The top plot shows the recorded sensor velocity components (red $-v_{x}$, blue $-v_{y}$ ) and the bottom plots show the velocity component error referenced to stage encoder feedback.

the measured tool $v_{x}$ and $v_{y}$ velocity components together with the overall tool speed for the path traversed in the highlighted rectangle in Figure $12 \mathrm{~b}$ ). Overlaid is the filtered signal processed using a 0.2 second mean averaging filter to remove robot vibrations and sensor noise. The results, showed tool speed variations of $\sim 25 \%$ when traversing corner radii, which resulted in wall height non-uniformities of $\sim 3 \mathrm{~mm}$ after five layers which could then be corrected by controlling the wire-feed rate used in the WAAM process [16].

\section{Conclusions}

A speckle correlation sensor for the measurement of robotic tool speed has been developed and the sensor performance has been assessed in the laboratory using a six degree of freedom high-precision motion stage system to simulate robot motions and sensor misalignments.

The sensor accuracy was found to be high with a maximum error of $\pm 0.01 \mathrm{~mm} / \mathrm{s}$ in mean recorded velocity over the full range $\pm 70 \mathrm{~mm} / \mathrm{s}$, over a lower velocity range of $\pm 10 \mathrm{~mm} / \mathrm{s}$, more typically used in the WAAM process the error is reduced to $<0.004 \mathrm{~mm} / \mathrm{s}$.

The effect of misalignment of the sensor on the robot was assessed for variation in both working distance and angular alignment. It was found that the sensor accuracy can be maintained to within $0.025 \mathrm{~mm} / \mathrm{s}(<0.04 \%)$ over a working distance variation of $\pm 5 \mathrm{~mm}$ from the sensor design distance over a $\pm 70 \mathrm{~mm} / \mathrm{s}$ measurement range, hence the sensor is robust to working height changes during operation or installation errors. For angular misalignments of the sensor, the degradation of sensor accuracy was more pronounced than for a variation in working distance, with bias error increasing to a maximum $\pm 0.4 \mathrm{~mm} / \mathrm{s}$ at $\pm 70 \mathrm{~mm} / \mathrm{s}(0.6 \%)$ for a misalignment of $5^{\circ}$. 

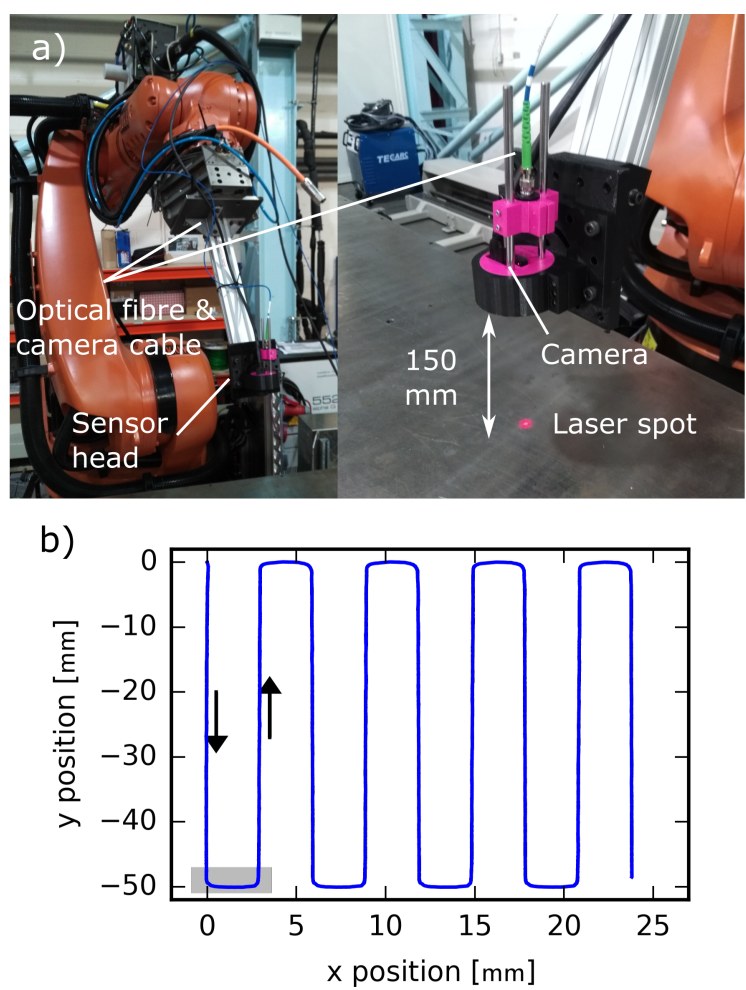

Figure 12: a) the speckle velocimetry sensor mounted on a Kuka KR150 robot. b) Oscillatory wall building path; arrows show direction of travel and highlighted rectangle showing the path traversed in Figure 13.

The sensor precision was assessed using a circular path giving continually varying $v_{x}$ and $v_{y}$ velocity components. Stage encoder velocity feedback data was used to determine the sensor precision by calculation of the instantaneous velocity error, with peak errors of $\pm 0.34 \mathrm{~mm} / \mathrm{s}$ at 1 $\mathrm{mm} / \mathrm{s}$ and $\pm 2.28 \mathrm{~mm} / \mathrm{s}$ at $70 \mathrm{~mm} / \mathrm{s}$ where the difference between the two speeds can be attributed to increased vibrations at higher stage velocities. This level of precision is found to be consistent with the peak fitting accuracy used in the signal processing and further improvement may be possible by improving the peak fitting process.

Finally an example of the sensors application to robotic manufacturing has been presented where the sensor was applied to tool speed measurement for path planning in the wire and arc additive manufacturing process[15, 16] using a KUKA KR150 L110/2 industrial robot.

\section{Acknowledgement}

This work was supported by the Engineering and Physical Sciences Research Council (EPSRC) UK [grant numbers EP/M020401/1, EP/N002520/1]. All data supporting this study are openly available from the Cranfield University repository at http://dx.doi.org/10.17862/cranfield.rd.4758472. 


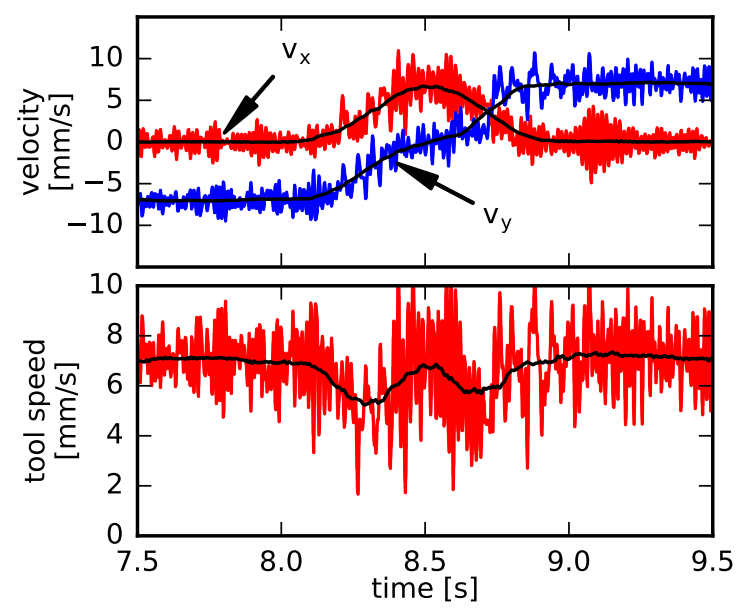

Figure 13: Measured robot velocity when traversing the corner shown in the highlighted rectangle in Figure 12 b). Top axis shows the $v_{x}$ (red) and $v_{y}$ (blue) velocity components and 0.2 second averaging filtered velocity components (solid lines). Bottom axis shows the combined tool speed indicating clear drops in the overall tool speed when traversing corner radii.

\section{Appendix A. Derivation of expression for accuracy achievable from calibration (equation} 4)

For a sensor with a true scaling factor $T$ and applied calibration translation, $a$ known to an accuracy $\Delta a$ then the calibrated scaling factor is:

$$
T^{\prime}=\frac{T(a \pm \Delta a)}{a}=T \pm \frac{T \Delta a}{a}
$$

The error in the determined scaling factor is given by $\Delta T=T^{\prime}-T$ substituting in A.1 and rearranging gives an expression for the scaling factor accuracy for a given calibration distance and accuracy:

$$
\frac{\Delta T}{T}= \pm \frac{\Delta a}{a}
$$

To determine the magnitude of the resulting velocity error resulting from this calibration error, the simple case without cross-sensitivity to other components is assumed where only a single scaling factor is involved. Given a true velocity, $v$ then the speckle shift in time interval, $d t$ is $A=T v d t$. If the scaling factor $T^{\prime}=T \pm \Delta T$ is then used, the measured velocity $v^{\prime}$ is given by:

$$
v^{\prime}=\frac{A}{T^{\prime}} \frac{1}{d t}=\frac{A}{(T \pm \Delta T)} \frac{1}{d t}=\frac{T v}{(T \pm \Delta T)}=\frac{1}{(1 \pm \Delta T / T)} v
$$

Finally the velocity error is given by $\Delta v=v^{\prime}-v$, substituting in A.2 and A.3 and rearranging gives the final expression for the velocity error for a given calibration distance and accuracy.

$$
\frac{\Delta v}{v}=\frac{1}{(1 \pm \Delta a / a)}-1
$$




\section{References}

[1] T. Brogårdh, Present and future robot control development: An industrial perspective, Annual Reviews in Control 31 (1) (2007) 69-79. doi : 10.1016/j . arcontrol.2007.01.002.

[2] B. Greenway, Robot accuracy, Industrial Robot: An International Journal 27 (4) (2000) 257-265. doi : 10.1108/ 01439910010372136.

[3] Y. Chen, F. Dong, Robot machining: Recent development and future research issues, International Journal of Advanced Manufacturing Technology 66 (9-12) (2013) 1489-1497. doi:10.1007/s00170-012-4433-4.

[4] B. Shirinzadeh, P. Teoh, Y. Tian, M. Dalvand, Y. Zhong, H. Liaw, Laser interferometry-based guidance methodology for high precision positioning of mechanisms and robots, Robotics and Computer-Integrated Manufacturing 26 (1) (2010) 74-82. doi:10.1016/j.rcim.2009.04.002.

[5] Z. Wang, L. Mastrogiacomo, F. Franceschini, P. Maropoulos, Experimental comparison of dynamic tracking performance of iGPS and laser tracker, International Journal of Advanced Manufacturing Technology 56 (1-4) (2011) 205-213. doi:10.1007/s00170-011-3166-0.

[6] C. Wang, W. Chen, M. Tomizuka, Robot end-effector sensing with position sensitive detector and inertial sensors, 2012 IEEE International Conference on Robotics and Automation (2012) 5252-5257doi : 10. 1109/ICRA . 2012. 6225180.

[7] G. Du, H. Shao, Y. Chen, P. Zhang, X. Liu, An online method for serial robot self-calibration with cmac and ukf, Robotics and Computer-Integrated Manufacturing 42 (2016) 39 - 48. doi : 10.1016/j.rcim.2016.05.006.

[8] L. Pérez, Í. Rodríguez, N. Rodríguez, R. Usamentiaga, D. García, Robot Guidance Using Machine Vision Techniques in Industrial Environments: A Comparative Review, Sensors 16 (3) (2016) 335. doi : 10. 3390/s16030335.

[9] J. Dainty, I The Statistics of Speckle Patterns, in: Progress in Optics, 1977, Ch. 1, pp. 2-46.

[10] I. Yamaguchi, Speckle Displacement and Decorrelation in the Diffraction and Image Fields for Small Object Deformation, Optica Acta: International Journal of Optics 28 (10) (1981) 1359-1376. doi:10.1080/713820454.

[11] D. Francis, T. O. Charrett, L. Waugh, R. P. Tatam, Objective speckle velocimetry for autonomous vehicle odometry, Applied optics 51 (16) (2012) 3478-90.

[12] T. O. Charrett, L. Waugh, R. P. Tatam, Speckle velocimetry for high accuracy odometry for a Mars exploration rover, Measurement Science and Technology 21 (2) (2010) 025301. doi : 10.1088/0957-0233/21/2/025301.

[13] M. Farsad, C. Evans, F. Farahi, Robust sub-micrometer displacement measurement using dual wavelength speckle correlation, Optics Express 23 (11) (2015) 14960. doi : 10.1364/OE.23.014960.

[14] R. Filter, T. Scharf, H. P. Herzig, High resolution displacement detection by speckle pattern analysis : accuracy limits in linear displacement speckle metrology, Journal of the European Optical Society: Rapid Publications 5 (2010) pp6. doi:10.2971/jeos.2010.10035s

[15] S. W. Williams, F. Martina, A. C. Addison, J. Ding, G. Pardal, P. Colegrove, Wire + arc additive manufacturing, Materials Science and Technology 0836 (March) (2015) 1743284715Y.000. doi:10.1179/1743284715Y. 0000000073.

[16] Y. K. Bandari, T. O. Charrett, F. Michel, J. Ding, S. W. Williams, R. P. Tatam, Compensation strategies for robotic motion errors for additive manufacturing (AM), in: International Solid Freeform Fabrication Symposium, Austin, Texas, 2016.

[17] J. P. Lewis, Fast normalized cross-correlation, in: Vision interface, Vol. 10, 1995, pp. 120-123. URL http://scribblethink.org/Work/nvisionInterface/nip.html

[18] P. Horváth, M. Hrabovský, P. Šmíd, Full theory of speckle displacement and decorrelation in the image field by wave and geometrical descriptions and its application in mechanics, Journal of Modern Optics 51 (5) (2004) 725742. doi: $10.1080 / 09500340408235548$

[19] T. O. Charrett, R. P. Tatam, Objective speckle displacement: an extended theory for the small deformation of shaped objects, Optics Express 22 (21) (2014) 25466. doi:10.1364/OE. 22 . 025466.

[20] M. Raffel, C. E. Willert, S. Wereley, J. Kompenhans, Particle Image Velocimetry: a practical guide, Experimental Fluid Mechanics, Springer Berlin Heidelberg, Berlin, Heidelberg, 2007. doi : 10.1007/978-3-540-72308-0.

[21] H. N. Nguyen, J. Zhou, H. J. Kang, A new full pose measurement method for robot calibration., Sensors (Basel, Switzerland) 13 (7) (2013) 9132-9147. doi:10.3390/s130709132.

[22] A. Nubiola, I. A. Bonev, Absolute calibration of an ABB IRB 1600 robot using a laser tracker, Robotics and Computer-Integrated Manufacturing 29 (1) (2013) 236-245. doi:10.1016/j.rcim.2012.06.004. 
2018-04-23

\section{A non-contact laser speckle sensor for the measurement of robotic tool speed}

Charrett, Thomas O. H.

Elsevier

Charrett TOH, Bandari Y, Michel F, Ding J, Williams SW, Tatam RP, A non-contact laser speckle sensor for the measurement of robotic tool speed, Robotics and Computer-Integrated Manufacturing, Volume 53, October 2018, pp. 187-196 http://dx.doi.org/10.1016/j.rcim.2018.04.007

Downloaded from Cranfield Library Services E-Repository 